visited by the GP who developed the tool and clinicians were trained in the use of it. It was then trialled for 8 weeks. In addition focus groups were held with 2 groups of young people $(n=25)$ to assess their opinion of the acceptability and utility of the THT.

Results 2 of the practices completed 51 THT between them. 1 practice did not complete any. The pilot centres who used the THT agreed it was useful and improved the focus on this age group in addition to improving communication. They commented it was feasible to use it within a 10 minute consultation and was also a useful training tool for junior staff. Young people were positive about a professional taking an interest in their health beyond the presenting complaint and agreed the topics covered were relevant and important.

Conclusions Primary care staff and young people confirmed that the THT is an important and useful assessment tool that can be used in primary care. Practices need support and training in how to use the tool and also confidence in what to do with the information uncovered - this requires knowledge about local resources and referral pathways and would benefit from the development of accompanying materials. Wider dissemination would potentially improve young people's experience of primary care leading to better utilisation in addition to the health benefits of earlier intervention and prevention where appropriate.

\section{G525(P) ABSTRACT WITHDRAWN}

\section{G526(P) ABSTRACT WITHDRAWN}

\section{G527(P) NEONATAL READMISSION RATES: AN AUDIT OF CURRENT PRACTICE}

R Seed, T Cullen, J Rajaram. Paediatrics, University Hospitals of Morecambe Bay NHS FT, Lancaster, UK

\subsection{6/archdischild-2020-rcpch.445}

Aims

- To identify the rate at which neonates (less than 28 days old) are admitted to the children's ward and the indication for admission.

- To highlight potentially avoidable causes for neonatal admissions, with a view to reducing them.

Methods We identified our population by comparing our local birth rate, and or neonatal admission rate to the children's ward. Secondly, we audited all neonatal admissions to the children's ward over a 12 week period. We reviewed electronic records and assessed 5 criteria:

- Reason for admission

- Source of referral

- Day of life of admission

- Duration of admission

- Whether admission potentially avoidable
Results Our local birth rate is 2,000 annually. This equates to approximately 450 livebirths over the 12 week period. 32 neonates out of 450 live births during this timeframe were admitted to the children's ward. A hospital admission rate of $7 \%$.

- Reason for admission:

$11(34 \%)$ suspected/confirmed sepsis

$10(31 \%)$ jaundice

8 (25\%) weight loss

$3(9 \%)$ other

- Source of referral:

$17(53 \%)$ community midwives

$6(19 \%)$ GP

$6(19 \%)$ A\&E

$3(9 \%)$ other

- Day of life on admission:

$7(21 \%)$ attended within the first 4 days of life

$8(25 \%)$ attended on day 5 of life

$8(25 \%)$ attended between days $6-15$ of life

$9(28 \%)$ attended between days $16-28$ of life

- Length of Stay:

$8(25 \%)$ stayed 1 night

$15(47 \%)$ stayed 2 nights

9 (28\%) stayed longer

- Admission avoidable?

$12(38 \%)$ were avoidable

$16(50 \%)$ were not avoidable

$4(13 \%)$ were not clearly avoidable or unavoidable

Conclusion $7 \%$ of babies are admitted to paediatrics in the neonatal period. $38 \%$ of admissions were avoidable. Almost all avoidable admissions are breastfed babies admitted with weight loss. Nearly all were referred by the community midwifery team on day 5 , co-inciding with newborn blood spot screening. Weight loss $>13 \%$ was common. All were discharged home within 2 days with a feeding plan.

We are now looking at how to support these families in the community to prevent avoidable neonatal admissions to paediatrics.

Source of referral:

\section{G528(P) HICKAM'S DICTUM, AND A CASE OF INFLAMMATORY BOWEL DISEASE IN THE CONTEXT OF PRE-EXISTING AVOIDANT-RESTRICTIVE FOOD INTAKE DISORDER}

DT Watkin. Department of General Paediatrics, Birmingham Women's and Children's NHS FT, Birmingham, UK

\subsection{6/archdischild-2020-rcpch.446}

Background 'Occam's razor' is a commonly-cited principle of diagnostic parsimony; that plurality should not be posited with necessity, and that a single unifying diagnosis is likely to be the best one. However, a counterargument can be expressed as 'Hickam's dictum'; that a patient may have as many diseases as he or she pleases.

Case We present a case of a 14-year-old girl who presented with weight loss. She had a background of severe anxiety with agoraphobia and emetophobia, with associated avoidantrestrictive food intake disorder. She was referred to the hospital by community mental health services for dietetic input and management of potential re-feeding syndrome.

Due to her anxiety, the patient wouldn't allow any blood tests or investigations. Engagement with dieticians, 